\title{
Astronomical x-ray optics using mono-crystalline silicon: high resolution, light weight, and low cost
}

William W. Zhang, Kim D. Allgood, Michael P. Biskach, Kai-Wing Chan, Michal Hlinka, et al.

William W. Zhang, Kim D. Allgood, Michael P. Biskach, Kai-Wing Chan, Michal Hlinka, John D. Kearney, James R. Mazzarella, Ryan S. McClelland, Ai Numata, Raul E. Riveros, Timo T. Saha, Peter M. Solly, "Astronomical xray optics using mono-crystalline silicon: high resolution, light weight, and low cost," Proc. SPIE 10699, Space Telescopes and Instrumentation 2018:

Ultraviolet to Gamma Ray, 1069900 (6 July 2018); doi: 10.1117/12.2312879

Event: SPIE Astronomical Telescopes + Instrumentation, 2018, Austin, Texas, United States 


\title{
Astronomical X-ray Optics Using Mono-crystalline Silicon: High Resolution, Light Weight, and Low Cost
}

\author{
William W. Zhang, Kim D. Allgood ${ }^{1}$, Michael P. Biskach ${ }^{1}$, Kai-Wing Chan ${ }^{2}$, \\ Michal Hlinka ${ }^{1}$, John D. Kearney ${ }^{1}$, James R. Mazzarella ${ }^{1}$, Ryan S. McClelland, \\ Ai Numata ${ }^{1}$, Raul E. Riveros ${ }^{2}$, Timo T. Saha, and Peter M. Solly ${ }^{1}$ \\ NASA Goddard Space Flight Center, Greenbelt, MD 20771 \\ ${ }^{1}$ also Stinger Ghaffarian Technologies, Inc., Greenbelt, MD 20770 \\ ${ }^{2}$ also University of Maryland-Baltimore County, Baltimore, MD 21250
}

\begin{abstract}
X-ray astronomy critically depends on X-ray optics. The capability of an X-ray telescope is largely determined by the point-spread function (PSF) and the photon-collection area of its mirrors, the same as telescopes in other wavelength bands. Since an X-ray telescope must be operated above the atmosphere in space and that X-rays reflect only at grazing incidence, X-ray mirrors must be both lightweight and thin, both of which add significant technical and engineering challenge to making an X-ray telescope. In this paper we report our effort at NASA Goddard Space Flight Center (GSFC) of developing an approach to making an Xray mirror assembly that can be significantly better than the mirror assembly currently flying on the Chandra $\mathrm{X}$-ray Observatory in each of the three aspects: PSF, effective area per unit mass, and production cost per unit effective area. Our approach is based on the precision polishing of mono-crystalline silicon to fabricate thin and lightweight X-ray mirrors of the highest figure quality and micro-roughness, therefore, having the potential of achieving diffraction-limited X-ray optics. When successfully developed, this approach will make implementable in the 2020s and 2030s many X-ray astronomical missions that are currently on the drawing board, including sounding rocket flights such as OGRE, Explorer class missions such as STAR-X and FORCE, Probe class missions such as AXIS, TAP, and HEX-P, as well as large missions such as Lynx.
\end{abstract}

Keywords: X-ray optics, lightweight optics, silicon mirror, meta-shell, x-ray optics

\section{INTRODUCTION}

The importance of X-ray optics has been recognized since before the discovery of extra-solar X-rays in the early 1960s [1]. In many ways, the history of X-ray astronomy is a history of developing better X-ray optics to enable more powerful X-ray observatories. "Better" means improvement in one or more of the three metrics: better point-spread-function (PSF) or higher angular resolution, larger effective area, and lower production cost. Any significant improvement in one or more of these three metrics typically has enabled a quantum leap in capability of X-ray observatories. The three currently operating X-ray telescopes, Chandra [2], XMM-Newton [3], and NuSTAR [4] represent the state of the art of X-ray mirror making. Chandra's mirror, made in the 1990s using the traditional "grind and polish" process and launched into space in 1999, has an exquisite angular resolution of 0.5 " HPD, but has an extremely small effective area of only $800 \mathrm{~cm}^{2}$ for its very large mass of approximately $1,500 \mathrm{~kg}$ and large volume. XMM-Newton's mirrors, contemporaries of the Chandra mirror, made of electro-formed nickel shells, has moderate angular resolution of 15" Half-PowerDiameter (HPD) with a moderately large effective area of $1,400 \mathrm{~cm}^{2}$ per module for a mass of $450 \mathrm{~kg}$. NuSTAR's mirrors, however, made in the late 2000s of slumped glass sheets, has a relatively poor angular resolution of 58" HPD, but has an enormous equivalent effective area of $2,400 \mathrm{~cm}^{2}$ for a very small mass of only $35 \mathrm{~kg}$ per module.

Space Telescopes and Instrumentation 2018: Ultraviolet to Gamma Ray, edited by Jan-Willem A. den Herder, Shouleh Nikzad, Kazuhiro Nakazawa, Proc. of SPIE Vol. 10699, 1069900 · C 2018 SPIE CCC code: $0277-786 X / 18 / \$ 18 \cdot$ doi: $10.1117 / 12.2312879$ 
An ideal X-ray mirror should have both good angular resolution and large effective area. It is generally the case, however, that angular resolution and effective area work against each other, as illustrated above by the three currently operational X-ray telescopes. As of the late 2010s, every X-ray astronomical mirror is a scientifically useful compromise of the three metrics: angular resolution, effective area, and production cost. The Holy Grail of X-ray astronomical optics development is to develop a process that can manufacture X-ray mirrors that have good angular resolution and large photon-collecting area at an affordable production cost. The objective of our Next Generation X-ray Optics team at NASA Goddard Space Flight Center is to develop such a technology.

We use a few guidelines to help us effectively accomplish this objective. First, we absorb the knowledge and lessons learned from designing, building, and testing past mirrors, including those of Einstein [5], ROSAT [6], BBXRT [7], ASCA [8], Suzaku [9], XMM-Newton [5], Chandra [2], Swift/XRT [10], and NuSTAR [4]. Each of these mirrors represented the pinnacle of technology of its own time under its unique scientific and budgetary circumstance. At least in principle we must devise a process that utilizes the knowledge and the lessons that have been accumulated from those past missions. Second, we must make the most of available technologies in industry, both in terms of technical equipment and materials, avoiding to the extent possible requiring one of a kind equipment that must be custom-designed and -built at enormous expense and risk obsolescence in a short time. Third, we must devise a process that is capable of, at least in principle, reaching diffraction-limited performance. Given the grazing incidence nature of X-rays and the concentric-shells design of an astronomical X-ray mirror assembly, the diffraction-limited performance can be practically taken to be approximately $0.1 " \mathrm{HPD}$ at $1 \mathrm{keV}$.

Developing an X-ray mirror-making process, from conception and maturity such that it can be used for implementing an X-ray telescope as part of a space observatory, is a circuitous, arduous, and expensive journey. Such a process must meet the many criteria that are imposed on any process that makes spaceflight hardware, including scientific performance, spaceflight worthiness, long term stability, not to mention contamination control and logistic feasibility under rigorous budgetary and schedule constraints. We have naturally divided our work into three distinct, yet highly related, components: technology, engineering, and production. They are distinct in that they each have their emphasis on meeting different aspects of the ensemble of requirements. They are highly related to each other in that they each in their own way impact and impose requirements on the others.

By "technology" we mean very basic technical elements that underlie our approach. In this specific case, it includes four elements: mirror fabrication, mirror coating, mirror alignment, and mirror bonding. By "engineering" we mean the design, analysis, and testing process that take the basic technical elements of the technology to arrive at a design for a mirror assembly that meet all requirements, including scientific performance such as PSF and effective area as well spaceflight worthiness such as structural integrity and longer term stability, contamination control, etc. Finally, by "production" we mean the manufacture process that implements the design, encompassing all the logistics necessary to meet the technical and programmatic requirements imposed on making an X-ray mirror assembly.

\section{TECHNOLOGY}

Mirror Fabrication Technology here means four technical elements: mirror fabrication, mirror coating, mirror alignment, and mirror bonding. Of these four elements, mirror fabrication is the foundation of everything else. Taking into account all kinds of considerations, including factors such as X-ray diffraction limits, desires for large effective area and lightweight and thin mirrors, engineering, and production logistics, we have arrived at the conclusion that the dimensions of the basic mirror element of our technology should be 
approximately $100 \mathrm{~mm}$ by $100 \mathrm{~mm}$ and by $0.5 \mathrm{~mm}$, as shown in Figure 1 . The material of the mirror segment should be mono-crystalline silicon $[11,12,13,14,15,16, \& 17]$.

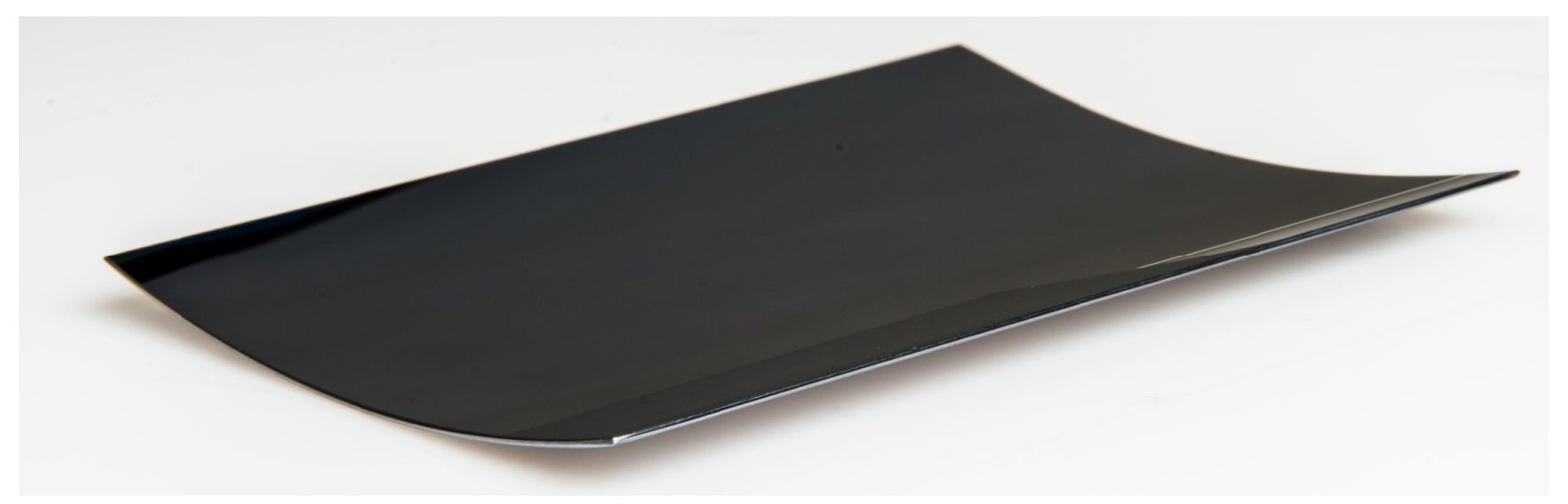

Figure 1. The basic element of our mirror technology: a mirror segment. Its typical dimensions are $100 \mathrm{~mm}$ by $100 \mathrm{~mm}$ by $0.5 \mathrm{~mm}$, made of mono-crystalline silicon. A typical X-ray mirror assembly is composed of many thousands of mirror segments of many different mathematical prescriptions like this one, coated, aligned, and bonded together.

Following the tradition of making high quality optics, we require that each mirror segment must be of sufficient quality in figure and micro-roughness to meet specifications derived from higher-level observatory requirements. In order to fabricate a mirror to meet the stand-alone figure requirements, it is necessary to use a material free of internal stress. The only commonly available such material is mono-crystalline silicon. This is fortuitously important because mono-crystalline silicon is the basic material for the semiconductor industry. As a result of enormous investment in the last five decades, very large blocks of mono-crystalline silicon is available at affordable prices. In particular a huge body of knowledge has been accumulated on how to handle, process, and machine mono-crystalline silicon.

The dimensions of the mirror segments are determined by performance and practical considerations. The 100 $\mathrm{mm}$ by $100 \mathrm{~mm}$ dimensions result from the dimensions of the prevailing $150 \mathrm{~mm}$ and $200 \mathrm{~mm}$ in diameter wafer sizes in the semiconductor industry. The thickness of $0.5 \mathrm{~mm}$ results from the combination of the desire to be many times thinner and lighter than Chandra's $25 \mathrm{~mm}$ thick mirror shells and the fact that the prevailing wafers are of similar thickness. As a result, we expect that an X-ray mirror assembly built out of the mirror segments like the one in Figure 1 will be approximately 50 times lighter than Chandra's mirror. Or equivalently and more importantly, given the same mass constraint as Chandra's mirror had, this technology will be able to build a mirror assembly that has approximately 50 times more photon-collecting area.

We have been developing a process to fabricate mirror segments [18]. As of early 2018, we are able to consistently fabricate mirror segments that have sub-arc-second figure quality, substantially similar to the quality of the Chandra mirror elements. The fabrication process consists of the following major steps. First, a block of mono-crystalline silicon, measured approximately $150 \mathrm{~mm}$ by $150 \mathrm{~mm}$ by $75 \mathrm{~mm}$ is procured and properly annealed to remove all residual internal stress. Then a CNC grinder is used to grind a conical approximation of the desired mirror segment's prescription onto the block. The ground surface is then lapped on a conical tool to impart a precise radius and a precise cone-angle to the conical surface, representing the zero-th and first order approximation to the mirror segment's mathematical prescription. The block is next mounted on a slicing machine to slice the precise conical form off of the block, resulting in a substrate approximately $1 \mathrm{~mm}$ in thickness and a residual block that is $1 \mathrm{~mm}$ thinner than before. The residual block is recycled for making the next substrate. The substrate is then etched in a standard HNA (a mixture of 
hydroflouric acid, nitric acid, and acetic acid) bath to remove damage to the crystal structure caused by the grinding and slicing operations, ending up with a substrate that is approximately $1 \mathrm{~mm}$ thick and has a precise radius and cone angle per mathematical prescription. This substrate is then mounted on a polishing machine for stress-polishing and smoothing to achieve both a good figure and good micro-roughness. During this process, the substrate, having a natural conical shape, is forced into a cylindrical shape and polished with colloidal silica slurry to remove all mid- to high-spatial-frequency errors. In practice, we have consistently found that all figure errors with a spatial wavelength shorter than $10 \mathrm{~mm}$ are completely removed whereas those errors with a spatial wavelength longer than $10 \mathrm{~mm}$ are substantially reduced, but not completely removed. The resulting mirror at this point of the process is typically 5" HPD (two reflections equivalent) and measures approximately $150 \mathrm{~mm}$ by $150 \mathrm{~mm}$ by $1 \mathrm{~mm}$. Because of the stress-polishing process, the mirror has severe roll-offs near the four edges. The mirror is then blocked on a mandrel and trimmed on four sides to remove those roll-offs and ground on the backside, i.e., the convex side to achieve the desired dimensions: $100 \mathrm{~mm}$ by $100 \mathrm{~mm}$ by $0.5 \mathrm{~mm}$. Both the trimming process and the grinding process cause severe surface and sub-surface damage to the crystal structure. As such, the mirror, with its polished surface protected, is etched again in an HNA bath to remove the damage, to recover its single-crystal nature.

The final step of the mirror segment fabrication is ion-beam figuring. Before the ion-beam figuring, the mirror has the desired dimensions and micro-roughness, but has a figure quality of approximately 5" HPD (2 reflections equivalent). On a typical ion-beam figuring machine, which is used in the semiconductor industry for trimming wafer thickness, a single pass improves the figure quality by a factor between 5 and 10, resulting in a mirror segment between 0.5 " and 1" HPD. Although not yet carried out, we expect that a second pass on the same ion-beam figuring machine will be able to improve the figure quality by another factor of several, bringing the mirror to approximately 0.2 " HPD, better than Chandra's mirror quality.

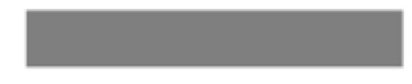

a) Bare silicon mirror

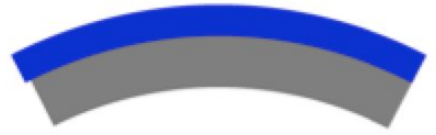

b) Silicon with oxide on onside

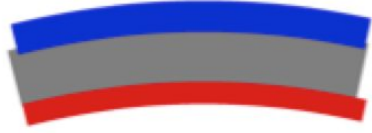

c) Coated with Ir

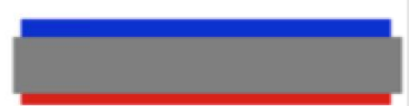

d) Oxide layer trimmed to balance Ir layer

Figure 2. An illustration of the coating process. a) A bare silicon mirror, undistorted. b) The same mirror with a layer of silicon oxide (blue) on one side that exerts a compressive stress, causing the mirror to distort. c) Now the same mirror is coated with a layer of iridium on the other side. The iridium's compressive stress cancels part of the compressive stress of the oxide layer. d) Precision trimming of the oxide layer thickness to balance the stress of the iridium layer, restoring the initial figure of the bare silicon mirror.

Mirror Coating Once a mirror segment is fabricated, it must be coated with another material to enhance its X-ray reflectivity. Although many different coatings are possible, traditionally a 30 -nm layer of the noble metal iridium is the best when all factors are considered. Unfortunately, $30 \mathrm{~nm}$ of iridium has significant stress and can severely distort the figure of a 0.5 -mm thick mirror. A way must be found to counter-balance or cancel the effect of this stress. In collaboration with MIT, we have developed a process that achieves essentially perfect stress cancellation [19]. The major steps of the process, shown in Figure 2, includes: growth of silicon oxide on both sides of the mirror, stripping of the oxide from the concave side, coating of the concave side with a thin layer of chromium as a binder and $30 \mathrm{~nm}$ of iridium, and carefully trimming the thickness of the oxide layer on the convex side to balance the stress of the iridium layer. As of June 2018, this 
stress-cancellation process has been exercised on two mirrors, resulting in less than 0.2 " HPD (two reflections equivalent) net distortion. There is good reason to believe that better metrology and more careful trimming of the oxide layer will reduce the net distortion by a factor of several, resulting in practically distortion-free coating.

Mirror Alignment Once a mirror segment is finished, both fabricated and coated, it needs to be precisely located and oriented to its prescribed configuration to contribute to the photon-collecting area of the mirror assembly [20]. For alignment, we utilize an important mathematical property of an X-ray mirror: four appropriately placed supports uniquely determine its location and orientation, as shown in Figure 3.

The locations of the four posts are optimized using finite element analysis. They can be optimized to minimize gravity distortion or they can be optimized to minimize frozen-in error once gravity is released in space. In principle, the two optimizations are different, but in practice the difference between them is small and can be neglected for now and will need to be taken into account when building and testing a $\sim 0.1$ " HPD mirror assembly in the future.

Once the locations of the four posts are determined, their radial heights determine the radial locations and alignment of the mirror segment. We have developed a systematic way to precision-lap the heights of these posts to a precision of approximately $0.1 \mu \mathrm{m}$ to align the mirror segment to better than 1" precision. The alignment process is an iterative process between Hartmann measurements to determine the orientation of the mirror and the deterministic precision lapping of the post heights.
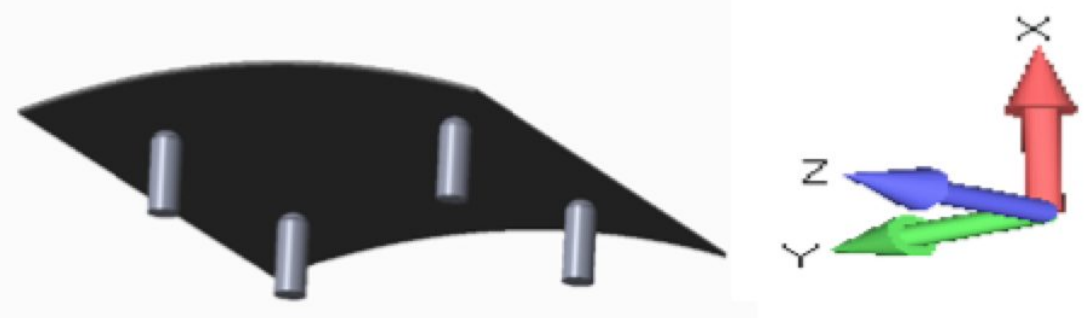

\author{
$\mathrm{X}-$ Gravity \\ $Y$ - Circumferential \\ Z-Optical Axis
}

Figure 3. An illustration of the principle of aligning a mirror segment. The mirror segment is supported at four points. The locations of the four points are optimized using finite element analysis to minimize gravity distortion and gravity release error.

Mirror Bonding Once a mirror is properly aligned; it must be permanently bonded to the posts. The process is a relatively simple one. Once the alignment is achieved and verified, the mirror segment is removed from the four posts. Then a small amount of epoxy is applied to the tip of each post and the mirror is replaced on the posts. Finally acoustic vibration from a boom box is applied to settle the mirror into its natural and relaxed aligned configuration.

Technology Validation by X-ray Testing The above four technical elements have been fully validated by repeatedly building and testing mirror modules, achieving expected X-ray images, as shown in Figure 4. These modules contain a single pair of mirror segments, a parabolic primary mirror and a hyperbolic secondary mirror, aligned and bonded on a robust silicon plate. The entire module, consisting of a thick silicon plate, eight posts, and a pair of mirrors, all made of mono-crystalline silicon, is completely athermal. It has been tested both at an X-ray beam line at NASA Goddard Space Flight Center and at the MPE's Panter X- 
ray Beam Line in Nureid, Germany. Both the measured X-ray image quality and measured effective areas at several different energies agree well with predictions based on optical surface metrology and theoretical expectations based on atomic scattering factors [21].
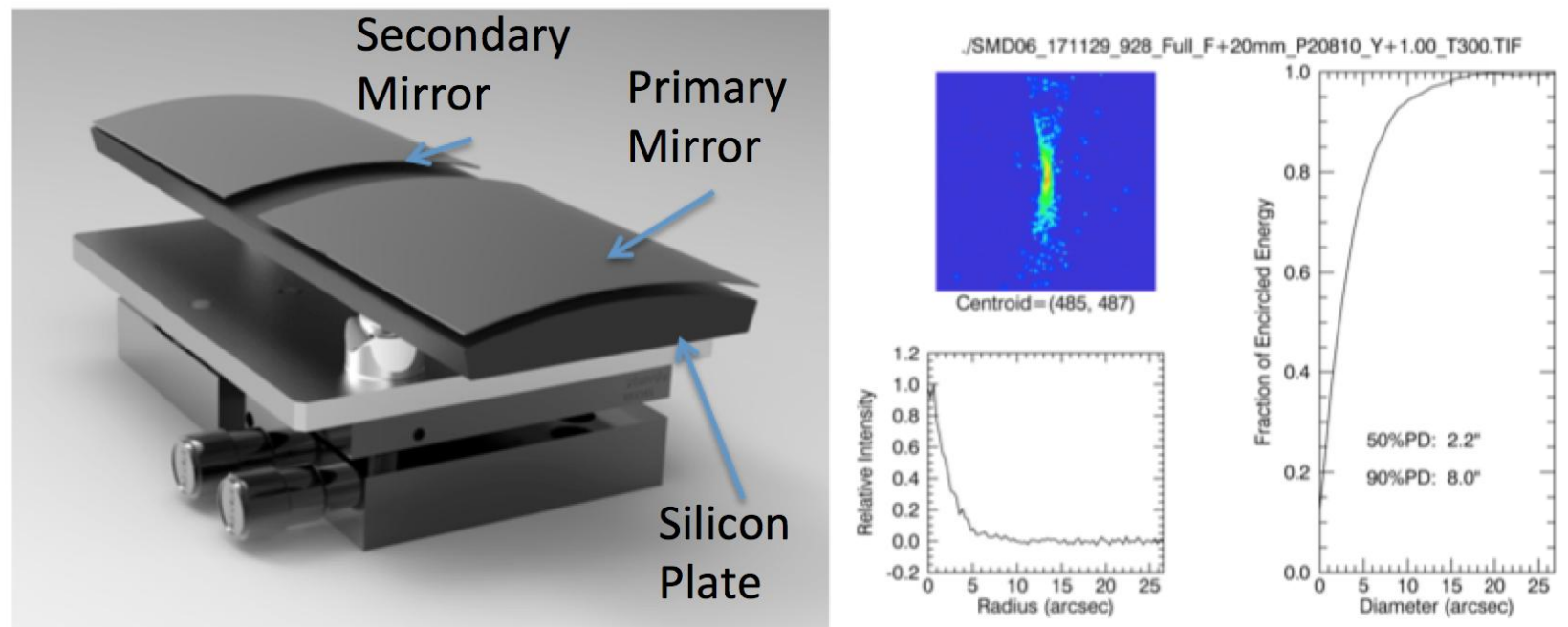

\section{Full illumination with Ti-K X-rays $(4.5 \mathrm{keV})$}

Figure 4. A pair of mirrors aligned, bonded, and tested in X-ray beams, achieving both expected X-ray images and effective areas. The image quality is 2.2 " HPD using $4.5 \mathrm{keV}$ X-rays. The measured effective area at $1 \mathrm{keV}$ is within $2 \%$ of the theoretical expected value. These measurements validate our entire technical approach.

\section{ENGINEERING}

Now that we have developed the four technical elements described in last section, as we continue to perfect them to achieve better angular resolution per the X-ray validation tests, we use them as the starting point to arrive at a design for implementing a practical mirror assembly that can pass the muster of spaceflight requirements. We have adopted a hierarchical approach to build a mirror assembly that will typically require many thousands of mirror segments for practical and logistical reasons. The approach is illustrated in Figure 5 [22].

The building-up of a mirror assembly is a two-step process. In the first step, the mirror segments are aligned and integrated into several, up to a dozen or so depending on the size of the mirror assembly, meta-shells. Each meta-shell is a collection of several hundred to several thousand mirror segments. Each meta-shell is composed of three components: the mirror segments, four posts for each segment, and a structural shell to which all mirror segments are bonded directly or indirectly. In practice these meta-shells can be built and tested sequentially, one after another, or they can be built and tested in parallel if required by schedule pressure. Then all the meta-shells are aligned and integrated together to form the mirror assembly.

Optical Design The approach can accommodate every possible X-ray astronomical telescope prescription. Each prescription is implemented by a combination of the mirror fabrication process and an appropriate displacement of the mirror segments to realize any principal surface such as called for by a WolterSchwarzschild design [23]. 
Stray Light Baffling The meta-shell approach is especially suited to implementing the most elaborate stray light baffles, essential requirements for some wide-field telescope designs. As shown in Figure 6, two types of stray light baffles are possible: cylindrical baffles in front of the primary mirrors and annular aperture stops that are attached to the backsides of mirror segments, as shown in Figure 6 [24].

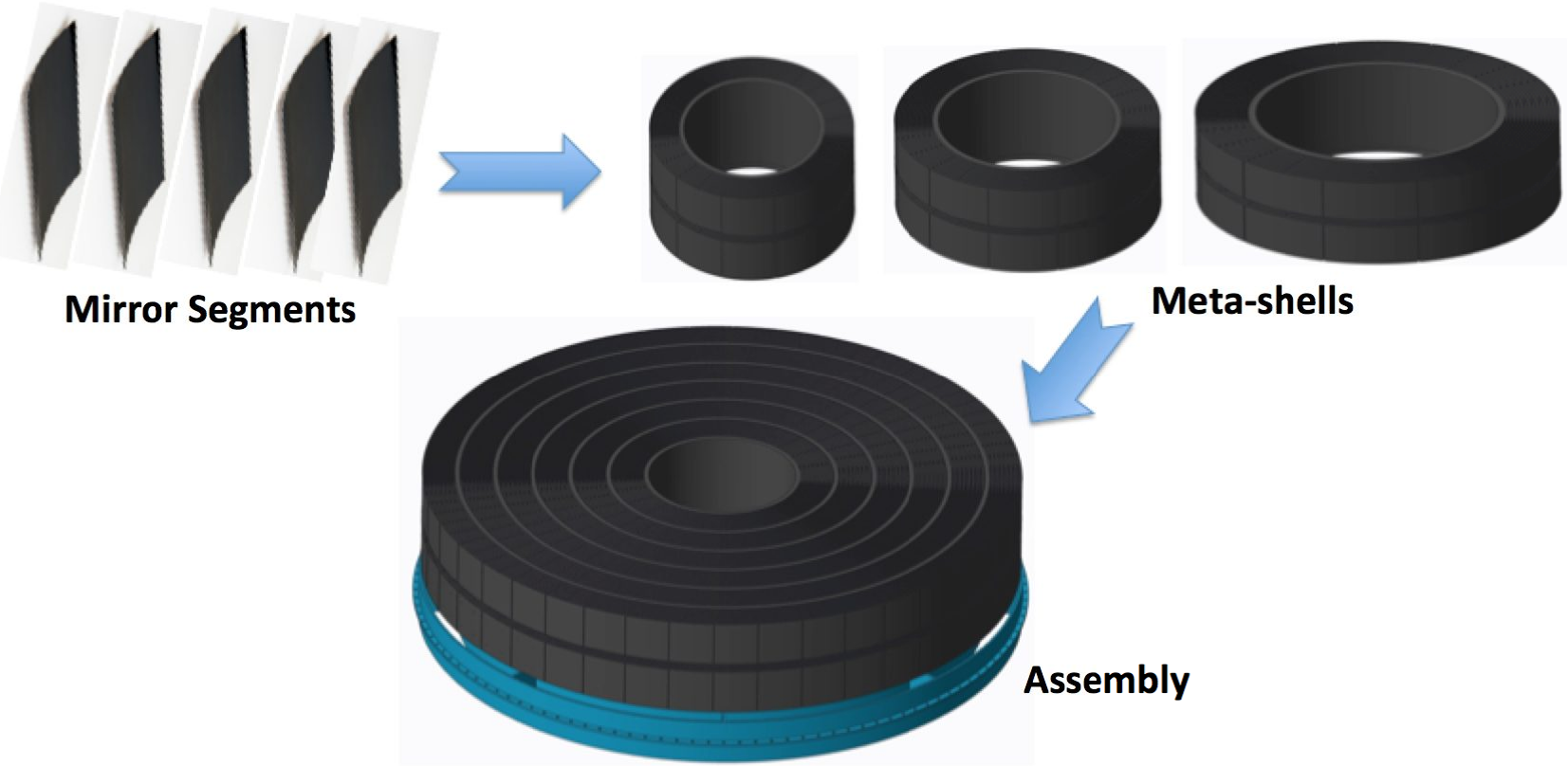

Figure 5. The hierarchical approach of building a large mirror assembly. The mirror assembly required for a typical future X-ray telescope has tens of thousands of mirror segments. They are first integrated into approximately 10 metashells each containing thousands of mirror segments. These meta-shells, in turn, are integrated into a final mirror assembly using standard engineering techniques such as flexures to isolate the meta-shells both structurally and thermally from the disturbance of the rest of the observatory.

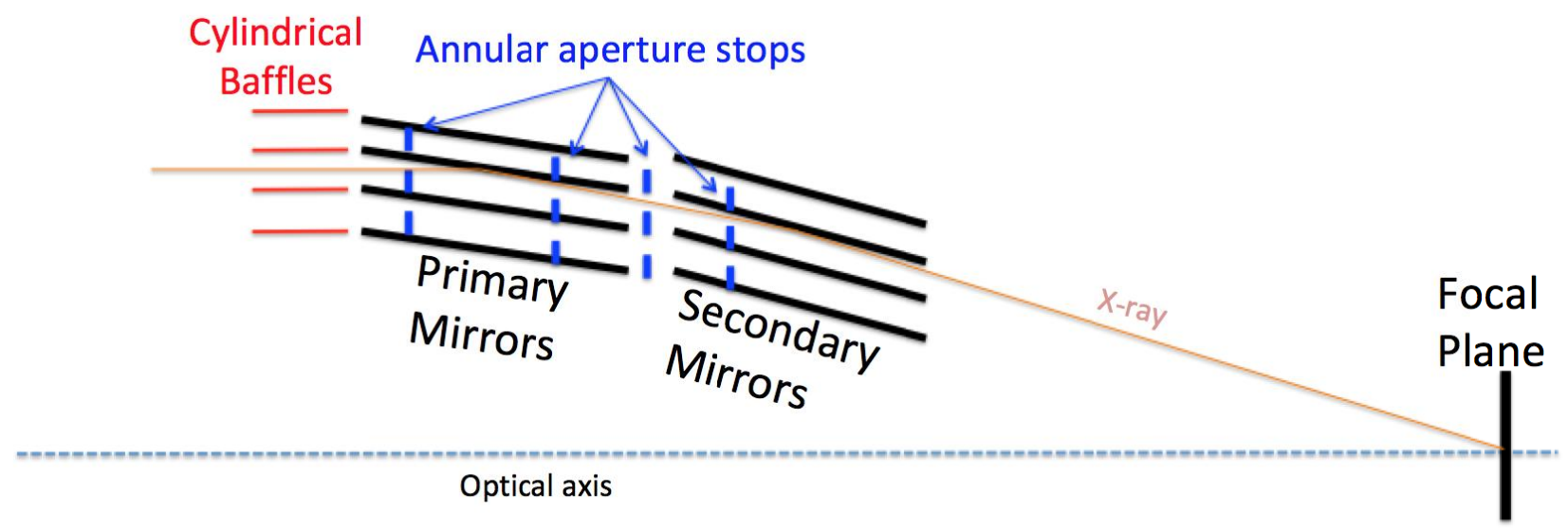

Figure 6. Two types of stray light baffles can be easily implemented in the meta-shell approach: the cylindrical baffles in front of the primary mirrors and the annular aperture stops between mirror segments. 
Structural Robustness Each meta-shell, consisting of a structural shell at its core and many lightweight mirror segments firmly attached to it, is a precision lightweight and stiff mechanical structure. As such, it is strong and can sustain the load typically associated with a rocket ride to space. We have done both finite element analysis and testing of specially designed engineering model to show that the meta-shell is indeed structurally sound [22]. As this technology is implemented for a specific mission where a specific rocket and observatory design is known, detailed analysis and testing will be necessary to further refine and validate the approach.

Thermal Design The mirror assembly and individual mirror segments must maintain excellent thermal equilibrium to preserve its optical performance. The excellent thermal conductivity and the fact the each meta-shell is made entirely of silicon except for a trace amount of other materials, such as iridium coating and epoxies, which have no thermal significance, facilitate the mirror assembly to achieve excellent thermal equilibrium. We have done thermal modeling of a generic mirror assembly in a low Earth orbit thermal environment and found that the thermal gradient across a typical mirror segment is of the order of 0.002 degrees C, sufficiently small to preserve the PSF to better than 0.2 " HPD.

Gravity Release Since each mirror segment, meta-shell, and the entire mirror assembly are, out of necessity, built and tested on the ground where gravity always exists, the mirror segment will necessarily change its figure upon entering space where gravity disappears. The four-point support of each mirror segment is the key to minimize gravity release error. We have done a preliminary analysis of a mirror assembly and found that PSF degradation of a mirror assembly made of $0.5 \mathrm{~mm}$ thick silicon mirrors is on the order of 0.2 " HPD, sufficiently small to be of concern at the present. We will revisit this issue when our technology has reached this level of PSF requirement.

\section{PRODUCTION}

Production starts when the technology and engineering are translated from knowledge on paper into reality, culminating in a mirror assembly passing all stringent performance and environmental tests and finally launched into space. In addition to the technological and engineering issues that have been addressed, the production must also address many practical issues of making and testing a mirror assembly, including schedule and cost which are at the heart of implementing every space flight mission.

As part of our technology development, we have avoided to the largest extent using custom-designed and built equipment. As such, the four technical elements, i.e., mirror fabrication, costing, alignment, and bonding, use only commercially available off-the-shelf equipment and materials.

Mono-crystalline Silicon is abundantly and inexpensively available because of the semiconductor industry. In all likelihood, given the importance and continued growth of the semiconductor industry, the trend of everlower price of mono-crystalline silicon will continue.

Capital Equipment needed for making the silicon mirrors includes CNC machines, interferometers, and ionbeam figuring machines, all of which are available from many vendors. They can be ordered and delivered in a few weeks to a few months. These machines are all highly efficient and reliable.

Polishing Tools are unique in that they must follow mathematical prescriptions, but they are only unique in their cone-angles and radii. These tools, typically made of stainless steel, can be fabricated easily and inexpensively by any reasonably equipped commercial machine shops. For a typical future mission, hundreds of unique tools may be needed. These tools can be procured commercially in less than six months. 
Polishing Machines are simple and built out of easily available parts. The overall cost of each machine is of the order of a few thousand dollars. As such, hundreds of them, one for each tool, can be built quickly with any reasonable budget for implementing a space flight mission.

The most expensive part of the production is, of course, the labor needed to perform all the tasks. Given the highly repetitive nature of the production process, we believe that labor can be saved as the technical process becomes more mature and streamlined and, therefore, more efficient. Since the making of a mirror assembly is just the repetition of the four technical elements for tens of thousands of times, it takes no leap of faith to realize that the efficiency and reliability of the four technical elements directly impact the overall production schedule and cost. It is our plan to mature and indeed to perfect each of the four technical elements, not only in making mirrors of the highest scientific performance such as PSF, but also in lowering the cost and minimizing the schedule.

\section{PROSPECTS}

We have outlined and reported our approach to build next generation X-ray telescopes. Our approach follows the traditional approach of "fabricate and assemble" where each component and each step is adequately measured and qualified such that the final mirror assembly is a precise sum of many precise parts. As such, the entire mirror assembly is free of stress to ensure its long-term stability. Perhaps most importantly this approach has the potential of making diffraction-limited X-ray optics in the next decade. As of 2018, we have demonstrated that this approach is capable of implementing a 5" telescope, likely even a 3" telescope, such as OGRE [25], STAR-X [26], FORCE [27], TAP [28], and HEX-P [29]. We expect this approach to make continued rapid progress in the coming years, culminating by the end of the 2020 s in demonstration that $\sim 0.1$ " telescopes can be built and flown, enabling missions currently on the drawing board, such as AXIS [30] and Lynx [31].

\section{ACKNOWLEDGEMENTS}

The authors wish to acknowledge the work of the many people who have helped advance this technology. Especially we want thank Dr. Vadim Burwitz and his team at the Panter X-ray Beam Facility at the Max Planck Institute for Extraterrestrial Physics in Garching, Germany, who conducted many X-ray tests and measurements of our mirror modules, helping us identifying problems and devising solutions. The work has been funded by NASA through the Astronomy \& Physics Research and Analysis Program (APRA) and the Strategic Astrophysics Technology Program under the Research Opportunities in Space and Earth Sciences (ROSES) program.

\section{REFERENCES}

[1] Giacconi, R. \& Rossi, B., “A telescope for soft X-ray astronomy,” J. Geophys. Res., Vol. 62, p. 773 (1960).

[2] VanSpeybroeck, L.P., “Performance Expectation versus Reality,” SPIE Proc. Vol. 3113, p. 89 (1997).

[3] Gondoin, P., et al., "X-ray multi-mirror (XMM) telescope," SPIE 2279, 86-100 (1994).

[4] Craig, W.W., et al., "Fabrication of the NuSTAR Flight Optics," SPIE Proc. Vol. 8147, p. 81470H-1 (2011).

[5] Young, P.S., "Fabrication of the High Resolution Mirror Assembly for the HEAO 2 Telescope," SPIE Proc. Vol. 194, p. 131 (1979).

[6] Aschenbach, B., "Design, Construction, and Performance of the ROSAT High-Resolution X-ray Mirror Assembly," Appl. Opt., Vol. 27, p. 1404 (1988).

[7] Serlemitsos, P.J., "Broad Band X-ray Telescope (BBXRT)," in “X-ray Astronomy in the 1980s," Ed. S.S. Holt, Proc. of a workshop held at NASA Goddard Space Flight Center, Greenbelt, MD, October 5-7 (1981). 
[8] Serlemitsos, P.J., “The X-ray Telescope Onboard ASCA,” PASJ, Vol. 47, pp. 105-114 (1995).

[9] Serlemitsos, P. J., et al. “The x-ray telescope onboard Suzaku," PASJ 59, 9-21 (2007).

[10] Burrows, D.N., et al., "Swift X-ray Telescope," SPIE Proc. Vol. 4140, p. 64 (2000).

[11] Zhang, W.W., et al., "Next generation X-ray optics: high-resolution, light-weight, and low-cost," A paper submitted to NASA in response to solicitation NNH11ZDA018L (2011).

[12] Zhang, W.W., et al., "Next generation astronomical X-ray optics: high angular resolution, light weight, and low production cost," SPIE Proc., 8443 (2012).

[13] Zhang, W. W., et al., "High Resolution and High Throughput X-ray Optics for Future Astronomical Missions," SPIE Proc., Vol. 8861, 88610N-1, (2013).

[14] Zhang, W. W., et al., "Affordable and Lightweight High-Resolution X-ray Optics for Astronomical Missions," SPIE Proc., Vol. 9144, 914415-1, (2014).

[15] Zhang, W.W., et al., “Toward Diffraction-Limited Lightweight X-ray Optics for Astronomy,” SPIE Proc, Vol. 9603 96030Q-1 (2015).

[16] Zhang, W.W., et al., "Lightweight and High-Resolution Single Crystal Silicon Optics for X-ray Astronomy," SPIE Proc., 9905 99051S-1 (2016).

[17] Zhang, W.W., et al., "Monocrystalline silicon and the meta-shell approach to building X-ray astronomical optics," SPIE Proc., Vol. 10399, p. 103990S-1 (2017).

[18] Riveros, R.E., et al., "Fabrication of lightweight silicon $x$-ray mirrors for high resolution $x$-ray optics," in these proceedings.

[19] Yao, Y., et al., "Thermal oxide patterning method for compensating coating stresses on silicon X-ray telescope mirrors," in these proceedings (2018)

[20] Chan, K.W., et al., "Alignment and Bonding of Silicon Mirrors for High-Resolution Astronomical X-ray Optics," in these proceedings.

[21] Saha, T.T., et al., "Analysis of the NGXO telescope x-ray Hartmann data," in these proceedings.

[22] McClelland, R.S., et al., "Design and analysis of an x-ray mirror assembly using the meta-shell approach," SPIE Proc., Vol. 9905 (2016)

[23] Saha, T.T., "General surface equations for glancing incidence telescopes,” Appl. Opt., Vol. 26, p. 658 (1987).

[24] Moran, E.C. \& Harvey, J.E., “Ghost Image Behavior In Wolter Type I X-Ray Telescopes,” SPIE Proc., 0830, 254 (1988).

[25] Tutt, J., et al., "The Off-plane Grating Rocket Experiment (OGRE) system overview," in these proceedings (2018).

[26] McClelland, R.S., et al., "The STAR-XX-ray Telescope Assembly," SPIE Proc., Vol. 10399 (2017).

[27] Nakazawa, K., et al., "The FORCE mission: science aim and instrument parameter for broadband X-ray imaging spectroscopy with good angular resolution," in these proceedings (2018).

[28] Astrophysical Transient Probe (ATP) website: https://asd.gsfc.nasa.gov/tap/

[29] Madsen, K., et al., "The high-energy X-ray probe," in these proceedings (2018)

[30] Mushotzky, R.F., et al., "AXIS: a probe class next generation high angular resolution $x$-ray imaging satellite," in these proceedings (2018).

[31] Gaskin, J.A., et al, "The Lynx X-ray observatory: concept study overview and status," in these proceedings. 